\title{
Editorial: layers at interfaces
}

\author{
Lucyna Holysz \\ Professor, The Faculty of Chemistry, Maria Curie-Sklodowska University, \\ Lublin, Poland
}

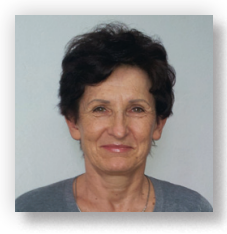

The meeting entitled Layers at Interfaces commemorates the 70th birthday of Prof. Emil Chibowski. It was held on May 17 and 18 at the Faculty of Chemistry, Maria Curie-Sklodowska University, Lublin, Poland. Prof. Chibowski has devoted nearly 50 years of his life to research on surface chemistry and molecular layers at interfaces. His biography follows this editorial.

Interfacial layers, having several more distinctive properties than contacting phases, play an essential role in stability and separation of multi-phase and colloidal systems and therefore, are vital to many technological processes and formulation of products, especially advanced materials and multiphase products. Control over molecular architectures at solid-liquid, solid-gas, liquidliquid and liquid-gas interfaces can be accomplished through adsorption or deposition (spreading, dip- and spin-coating, layerby-layer deposition, sputtering and others).

The papers presented at this meeting discussed in depth the science of surfaces and interfaces and covered a broad range of molecular layers at different interfaces. In all, 13 invited papers were presented, an abridged version of which will be published in Surface Innovations across three issues: six papers appear in this issue, six in a subsequent issue, and one in the first issue of 2015.

In this issue, the paper to appear first in the collection is by Kallay et al. ${ }^{1}$ from the Faculty of Science, University of Zagreb, Croatia. Kallay et al. discuss the anisotropy of surface potential for inorganic crystals. It is well known that different planes exhibit different structures and properties and that planes are connected electrically through the bulk of crystal, and thus a measure of surface potential is an apparent value resulting from the contribution of each individual plane. In some systems, the value of one of the planes may dominate the average surface potential. The authors discuss the origin of surface potential at a metal oxide surface using a surface complexation model and present numerical simulations for the analysis of surface potential for individual crystallographic planes.

Following Kallay et al., the paper by Krasowska et al. ${ }^{2}$ of Ian Wark Institute, University of South Australia, Australia, reviews atomic force microscopy (AFM) techniques for imaging soft, deformable fluid layers and structures, emphasising the challenges and artifacts in AFM imaging. This review highlights the most critical parameters for both contact and noncontact AFM imaging. The importance of correct loading force on the observation of image artefacts is illustrated through the example of how tip-sample interactions influence the appearance of liquid droplets and air bubbles on solid surfaces.

The next article by Oćwieja and Adamczyk ${ }^{3}$ from Jerzy Haber Institute of Catalysis and Surface Chemistry in Cracow, Poland, describes the formulation and use of charge-stabilised silver particle suspensions synthesised by chemical reduction methods using various organic and inorganic reducing agents. Physicochemical characterisation of formulated nanoparticles, including determination of particle size distribution, hydrodynamic diameter, specific surface area, diffusion coefficient, electrophoretic mobility, and charge density, are discussed. The deposition of formulated monodisperse silver nanoparticles $(13-17 \mathrm{~nm})$ into high-density monolayers on a mica substrate using a diffusion-controlled selfassembly process is described. The nanoparticle deposition kinetics is analysed by the random sequential adsorption model.

The determination of solid surface free energy through contact angle measurement is described by Jańczuk and Zdziennicka ${ }^{4}$ from the Faculty of Chemistry, Maria Curie-Sklodowska University, Poland. The authors stress the need to account for pressure of surface and interfacial films formed by either adsorption or liquid spreading in the Young's equation and calculation of surface free energy. They further state that the surface and interfacial film characteristics of solids in contact with liquids may explain, in part, the differences between experimental solid surface free energy values obtained from contact angle measurements and those obtained with other methods or calculated theoretically.

The fabrication of hybrid multilayers composed of polyelectrolytes (PEs) and Prussian blue (PB) nanoparticles by layer-by-layer deposition is demonstrated by Warszyński et al. ${ }^{5}$ from Jerzy Haber Institute of Catalysis and Surface Chemistry, Poland. The study showed that a larger amount of PB nanoparticles could be adsorbed if the layers of conductive polymers (polypyrrole and polyaniline) were incorporated into a composite structure. A quartz crystal microbalance with dissipation was used to monitor the build-up of PE/PB multilayers. Due to the electrocatalytic activity of PB towards hydrogen peroxide, the use of cyclic voltamperometry was convenient to monitor the deposition process.

The contribution from Dynarowicz-Latka ${ }^{6}$ of the Faculty of Chemistry, Jagiellonian University, Poland, is the final paper in this issue. Dynarowicz-Latka investigated antitumour lipids (ATLs) 
in biomembranes. Langmuir monolayers were used as model membranes. Synthetic analogues of lysophosphatidylcholine, due to their anticancer properties, were used as a target drug. The transport of ATLs to the cells, which has not been well described in literature, is the focus of this study. The paper gives evidence of a successful application of Langmuir monolayers for the investigation of complex biological issues.

Liposomes, micelles, nanoparticles, single-walled carbon nanotubes, and dendrimers in aqueous solutions are promising carriers for systemic delivery of antitumour drugs such as doxorubicin (DOX). In a review article, Matyszewska ${ }^{7}$ from the Faculty of Chemistry, University of Warsaw, Poland, presents different types of drug delivery systems (DDS) to transport an anthracycline cytostatic antibiotic. This study has pointed out the present lack of efficient and safe DDS-DOX conjugates for cancer treatment despite numerous investigations carried out in the past 20 years.

The manipulation of surface topography of hydrophobic and hydrophilic materials remains a favorite area of study for numerous researchers in surface chemistry discipline. Drelich of the Department of Materials and Engineering, Michigan Technological University, USA and Marmur of the Department of Chemical Engineering, Technion-Israel Institute of Technology, Israel, offer a review paper on superhydrophobic and superhydrophilic surfaces and coatings. ${ }^{8}$ This review summarises the physics behind static contact angles and control over liquid droplet behaviour on smooth and rough surfaces. The review also covers several commercially available superhydrophobic and superhydrophilic coatings used in the production of antifogging screens, windows and lenses, antifouling coatings, microfluidic devices, biocompatible implant devices, coatings for enhanced boiling heat transfer, foils for food packaging, and many others.

The work of Dokowicz and Nowicki ${ }^{9}$ from the Faculty of Chemistry, Adam Mickiewicz University, Poland, is a piece of original research. The study analysed the morphology of liquid droplets deposited on a solid surface having narrow strips and grooves of triangular cross section. The behaviour of different volume droplets that settled on a defined solid surface was simulated using a gradient descent method and the Surface Evolver program to minimise the surface and interfacial energy of the system. The ratio of droplet dimension to groove size dictates the droplet shape on such symmetrically shaped rough surfaces. The authors further demonstrate nonmonotonic changes in shape for liquid droplets caused by the triangular grooves.

Zawala et al. ${ }^{10}$ of Jerzy Haber Institute of Catalysis and Surface Chemistry, Poland, studied the influence of an air entrapment with a porous structure made of a hydrophilic material immersed in water on the kinetics of air bubble collisions and their bouncing. A high-speed video recording, supported by numerical simulation, of air bubble bouncing off entrapped air bubbles revealed that both amplitude of bouncing and time of bubble immobilisation are affected by the air trapped in the porous surface with which the air bubble collides. Faster immobilisation of the bubble was due to a quicker drainage and rupture of foam (water) film.

Barczak et al. ${ }^{11}$ of the Faculty of Chemistry, Maria CurieSklodowska University, Poland, investigated changes in surface structure, porosity, and chemistry of three groups of carbon materials: ordered mesoporous carbon, glassy carbon, and multiwalled carbon nanotubes. Carbon-based materials were oxidised and subsequently functionalised with aminosilanes to improve material properties and extend their applications. Upon structural analysis of carbon materials, the authors found that the porous structure of carbon nanotubes remained unchanged, whereas the structure of mesoporous carbon collapsed during oxidation and functionalisation. On the other hand, the micropores of glassy carbon opened up during oxidation, but are unfortunately blocked by amino groups during functionalisation.

The article by Wilk et al. ${ }^{12}$ of the Faculty of Chemistry, Wroclaw University of Technology, Poland, describes a homologous series of dicephalic surfactants $\left(\mathrm{C}_{n} \mathrm{MEDA}\right)$ and their application towards improving the stability of solid nanoparticles of lipids (SLNs) and nanostructured lipid carriers (NLCs). Differential scanning calorimetry showed that stabilisation was accompanied by lowering the phase transition temperature, enthalpy of fusion, and recrystallisation index. The SLNs and NLCs stabilised by dicephalic surfactants constituted more stable products compared with those stabilised by their linear counterparts.

Although silica materials are used in a wide range of industrial and consumer applications due to their physicochemical properties, exposure to silica-containing dust has been associated with the risk of developing life-threatening lung diseases. To reduce the toxicity of silica, a phospholipid coating (comprising mostly DPPC) was proposed. Szczes and Holysz ${ }^{13}$ of the Faculty of Chemistry, Maria Curie-Sklodowska University, Poland, studied the effect of phospholipid (DPPC) mono- or bilayers on the surface properties of silica particles, as well as the effect of phospholipase $\mathrm{A}_{2}\left(\mathrm{PLA}_{2}\right)$ enzyme. The study has found that calcium ions affect DPPC adsorption on silica particles, and the $\mathrm{PLA}_{2}$ can remove lipid coatings.

I hope readers find all the 13 papers interesting to read. I welcome your reflections and comments on their content.

\section{REFERENCES}

1. Kallay, N.; Preočanin, T.; Sapunar, M.; Namjesnik, D. Common surface potential of two different crystal planes. Surface Innovations 2014, 2(3), 142-150.

2. Krasowska, M.; Niecikowska, A.; Beattie, D. A. Challenges in imaging of soft layers and structures at solid surfaces using 
atomic force microscopy. Surface Innovations 2014, 2(3), 151-159.

3. Oćwieja, M.; Adamczyk, Z. Monolayers of silver nanoparticles obtained by chemical reduction methods. Surface Innovations 2014, 2(3), 160-172.

4. Jańczuk, B.; Zdziennicka, A. Importance of surface layers in solid surface free energy determination. Surface Innovations 2014, 2(3), 173-183.

5. Pajor-Świerzy, A.; Kolasińska-Sojka, M.; Warszyński, P. Polyelectrolyte films with Prussian blue nanoparticles and conductive polymers. Surface Innovations 2014, 2(3), 184-193.

6. Dynarowicz-Łątka, P. Antitumor lipids in biomembranes modeled with the Langmuir monolayer technique. Surface Innovations 2014, 2(3), 194-200.

7. Matyszewska, D. Drug delivery systems in the transport of doxorubicin. Surface Innovations 2014, http://dx.doi. org/10.1680/si.13.00040.

8. Drelich, J.; Marmur, A. Physics and applications of superhydrophobic and superhydrophilic surfaces and coatings. Surface Innovations 2014, http://dx.doi.org/10.1680/ si.13.00017.

9. Dokowicz, M.; Nowicki, W. Morphology of a droplet deposited on a strip and triangular groove. Surface Innovations 2014, http://dx.doi.org/10.1680/si.13.00034.

10. Zawala, J.; Kosior, D.; Malysa, K. Air-assisted bubble immobilization at hydrophilic porous surface. Surface Innovations 2014, http://dx.doi.org/10.1680/si.13.00031.

11. Michalak-Zwierz, K.; Pietras-Ożga, D.; Gdula, K., et al. Surface modification of carbon nanomaterials by aminopropyltriethoxysilane. Surface Innovations 2014, http:// dx.doi.org/10.1680/si.13.00041.

12. Wiercigroch, K.; Lewińska, A.; Wilk, K. A. Multifunctional di-N-oxide surfactants in fabrication of lipid nanoparticles. Surface Innovations 2014, http://dx.doi.org/10.1680/ si.13.00038.

13. Szczes, A.; Holysz, L. Influence of DPPC layers and PLA on surface properties of silica particles. Surface Innovations 2014, http://dx.doi.org/10.1680/si.13.00027. 